\title{
Ventilação mecânica: avaliando o conhecimento dos enfermeiros em uma unidade de terapia intensiva em Porto Velho-RO
}

\author{
Mechanical ventilation: evaluating nurses' knowledge in an intensive care unit in Porto \\ Velho-RO
}

Ventilación mecánica: evaluando el conocimiento de los enfermeros en una unidad de terapia intensiva en Porto Velho-RO

Jaciene Oliveira da Silva ${ }^{1 *}$, Maria Edna de Oliveira ${ }^{1}$, Tathiane Souza de Oliveira ${ }^{1}$

\section{RESUMO}

Objetivo: Descrever o conhecimento do enfermeiro frente as práticas assistenciais ao paciente em uso de ventilação mecânica em uma Unidade de Terapia Intensiva em um hospital estadual no município de Porto Velho/RO. Materiais e Métodos: Optou-se por uma pesquisa de campo, com objetivo descritivo, com abordagem quantitativa. $\mathrm{O}$ instrumento utilizado foi um questionário com perguntas fechadas, aplicado aos enfermeiros. Resultados: A amostra final foi composta por 06 enfermeiros atuantes no Hospital e Pronto Socorro João Paulo II e 14 enfermeiros na AMI (Unidade de Assistência Médica Intensiva) totalizando uma amostragem de 20 enfermeiros. Conclusão: Este estudo destacou que o conhecimento científico, adquirido por cursos, capacitações, atualizações, ou seja, atividades de educação continuada ou permanente são estratégias podem e devem ser adotadas para melhoria do atendimento ao paciente em ventilação artificial.

Palavras-chave: Enfermeiro, UTI, Ventilação Mecânica.

\begin{abstract}
Objective: To describe nurses' knowledge regarding patient care practices using mechanical ventilation in an Intensive Care Unit at a state hospital in the city of Porto Velho / RO. Materials and Methods: We chose a field research, with a descriptive objective, with a quantitative approach. The instrument used was a questionnaire with closed questions, applied to nurses. Results: The final sample consisted of 06 nurses working in the Hospital and Emergency Room João Paulo II and 14 nurses in the AMI (Intensive Care Unit), with a sample of 20 nurses. Conclusion: This study emphasized that the scientific knowledge acquired by courses, training, updates, that is, activities of continuing or permanent education are strategies can and should be adopted to improve patient care in artificial ventilation.
\end{abstract}

Keywords: Nurse, UTI, Mechanical ventilation.

\section{RESUMEN}

Objetivo: Describir el conocimiento del enfermero frente a las prácticas asistenciales al paciente en uso de ventilación mecánica en una Unidad de Terapia Intensiva en un hospital estadual en el municipio de Porto Velho / RO. Materiales y Métodos: Se optó por una investigación de campo, con objetivo descriptivo, con abordaje cuantitativo. El instrumento utilizado fue un cuestionario con preguntas cerradas, aplicado a los enfermeros. Resultados: La muestra final fue compuesta por 06 enfermeros actuantes en el Hospital y Pronto Socorro Juan Pablo II y 14 enfermeros en la AMI (Unidad de Asistencia Médica Intensiva) totalizando un muestreo de 20 enfermeros. Conclusión: Este estudio destacó que el conocimiento científico, adquirido por cursos, capacitaciones, actualizaciones, o sea, actividades de educación continuada o permanente son estrategias que pueden y deben ser adoptadas para mejorar la atención al paciente en ventilación artificial.

Palabras clave: Enfermería, UTI, Ventilación Mecánica.

1União das Escolas Superiores de Rondônia (UNIRON), Porto Velho - RO. *E-mail: jacieneenfermeira@gmail.com 


\section{INTRODUÇÃO}

A ventilação mecânica (VM) é uma máquina que exerce uma função total ou parcial à atividade ventilatória, sendo um método de suporte para o tratamento de pacientes com insuficiência respiratória aguda ou crônica, com o propósito de melhorar o equilíbrio entre a oferta e a busca de oxigênio e aliviar o esforço respiratório dos clientes (NÓBREGA MML, 2014).

A Unidade de Terapia Intensiva (UTI) é uma unidade que recebe pacientes em estado crítico que carecem de cuidado adequado, com a finalidade de buscar reverter sua situação clínica, ampliando as possibilidades de recuperação e sobrevivência dos pacientes, sendo um ambiente que exige muita atenção da equipe de enfermagem (POLKEY E, MOXHAM DF, 2015).

O cuidado aos clientes em uso de ventilação mecânica torna-se um comprometimento dos enfermeiros, pois o progresso positivo dos pacientes advém da assistência continuada, os enfermeiros são aptos a oferecer a identificação de problemas que alcancem exatamente suas necessidades prestando um cuidado de excelência, com isso é preciso que os mesmo possuem uma vasta compreensão dos conceitos da VM (NÓBREGA MML, 2014).

O enfermeiro que exerce suas atribuições na Unidade de Terapia Intensiva deve estar capacitado a realizar esses cuidados aos pacientes graves, portanto observa-se que o enfermeiro está distanciando-se desse suporte ventilatório, provavelmente pelas várias funções que lhe são providas, ou podendo haver outro profissional realizando essa assistência (DAMASCENO FD et al., 2015).

Observa-se, atualmente, que os enfermeiros, estão ficando cada vez mais distantes do suporte ventilatório, talvez pelas inúmeras atribuições, ou por haver outro profissional fazendo esse tipo de assistência. É importante que os enfermeiros estejam preparados a prestar cuidados como realizar monitorização dos parâmetros ventilatórios e dos alarmes e controle das condições hemodinâmicas do paciente, visando a minimizar os efeitos adversos, quando necessário (CARRARETTO AR, 2014).

A assistência aos pacientes sob VM torna-se responsabilidade dos enfermeiros, como membro integrante da equipe da UTI, que está diretamente envolvido no processo do cuidado dos pacientes. Sendo que a evolução positiva destes depende de cuidados contínuos, capazes de promover a identificação de problemas que atinjam suas necessidades. Nesse contexto, o enfermeiro deve estar preparado e capacitado para tal finalidade, já que esses pacientes se encontram em estado grave (SILVA LCC, 2015).

O profissional tem o comprometimento de executar suas atribuições de sua profissão com caráter humano, científico e ético e, para alcançar esse propósito, é preciso ter apreciação ética, qualificação constante e competência de conhecimento moral para a conquista de medidas éticas no cumprimento profissional, portanto esse tema é intenso e bem debatido por inúmeros autores (CARRARETTO AR, 2014).

O estudo se propõe a responder a seguinte questão norteadora: Qual o conhecimento do enfermeiro no cuidado assistencial ao paciente em uso de ventilação mecânica? O presente estudo tem como objetivo descrever o conhecimento do enfermeiro frente as práticas assistenciais ao paciente em uso de ventilação mecânica em uma Unidade de Terapia Intensiva em Porto Velho/RO.

\section{MÉTODOS}

Estudo de natureza descritiva e bibliográfica com abordagem quantitativa. O estudo foi composto por enfermeiros que estavam de plantão na UTI Adulto. A amostra da pesquisa foi constituída por 20 enfermeiros que aceitaram participar da pesquisa. A pesquisa foi realizada no Hospital e Pronto Socorro João Paulo II e na AMI (Unidade de Assistência Médica Intensiva). Os critérios de inclusão foram estabelecidos da seguinte forma: foram incluídos enfermeiros atuantes na UTI, sendo homens e mulheres sem faixa etária na UTI adulta do Hospital e Pronto Socorro João Paulo II e da AMI do município de Porro Velho-RO e que assinaram o Termo de Consentimento Livre Esclarecido. Foram excluídos os enfermeiros em período de férias, licença ou afastamento durante o período da pesquisa, ausência em todas as tentativas de busca do profissional e os que não assinarem o TCLE. 
O estudo foi desenvolvido obedecendo ao estabelecido na Resolução n.․ 466/2012/CNS/MS. Em virtude de se tratar de uma pesquisa com seres humanos, o estudo foi submetido ao Comitê de Ética em Pesquisa (CEP) e após parecer favorável, iniciou-se a coleta de dados. A pesquisa foi realizada através da aplicação de um questionário elaborado pelas pesquisadoras deste estudo que contemplará 10 perguntas fechadas, relacionada a descrever o conhecimento do enfermeiro frente as práticas assistenciais ao paciente em uso de ventilação mecânica em uma Unidade de Terapia Intensiva em um Hospital Estadual no município de Porto Velho-RO. O presente estudo foi submetido à apreciação do CEP da UNINORTE, o qual expediu parecer favorável sob o número o ํㅜ‥132.261 em 05 de fevereiro de 2019.

\section{RESULTADOS}

A amostra final foi composta por 6 enfermeiros atuantes no Hospital e Pronto Socorro João Paulo II e 14 enfermeiros na AMI (Unidade de Assistência Médica Intensiva) totalizando uma amostragem de 20 enfermeiros que aceitaram participar da pesquisa após assinatura do TCLE. No que diz respeito ao sexo dos participantes, o estudo revelou que $86 \%(n=18)$ são representados pelo sexo feminino e $14 \%(n=2)$ são do sexo masculino. Quanto a variável da faixa etária percebe-se uma maior concentração de 26 a 33 anos, representando $48 \%(n=9)$, com $25 \%(n=5)$ representando a faixa etária de 34 a 41 anos e 42 a 49 anos com $20 \%(n=4)$ de representatividade, a faixa etária de 18 a 25 anos contabilizou $7 \%$ da amostra $(n=2)$. Quanto as especializações temos um quantitativo de $53,6 \% \quad(n=15)$ dos enfermeiros com especialização, 28,6\% ( $n=4)$ que possuem mestrado e 17,8\% $(n=1)$ com doutorado (Tabela 1).

Tabela 1 - Caracterização do perfil dos enfermeiros atuantes no HPJPII e na AMI em Porto Velho, 2019.

VARIÁVEIS N $\quad$ \%

\section{SEXO}

Feminino

Masculino

\section{FAIXA ETÁRIA}

$\begin{array}{lll}18 \text { a } 25 \text { anos } & 2 & 7 \% \\ 26 \text { a } 33 \text { anos } & 9 & 48 \% \\ 34 \text { a } 41 \text { anos } & 5 & 24 \% \\ 42 \text { a } 49 \text { anos } & 4 & 21 \%\end{array}$

\section{CURSOS}

$\begin{array}{ccc}\text { Especialização } & 15 & 53,6 \% \\ \text { Mestrado } & 4 & 28,6 \% \\ \text { Doutorado } & 1 & 17,8 \%\end{array}$

\begin{tabular}{lll}
\hline Total & 20 & $100 \%$
\end{tabular}

Fonte: Silva JO Oliveira ME e Oliveira TS, 2019.

Em relação a Figura 1 evidenciou-se que $89 \%(n=18)$ dos enfermeiros relataram que receberam treinamento para atuar nesse setor e $11 \%(n=2)$ relatam que não tiveram esse treinamento. 
Figura 1 - Distribuição das respostas dos Enfermeiros quanto ao recebimento do treinamento na UTI, Porto Velho/2019.

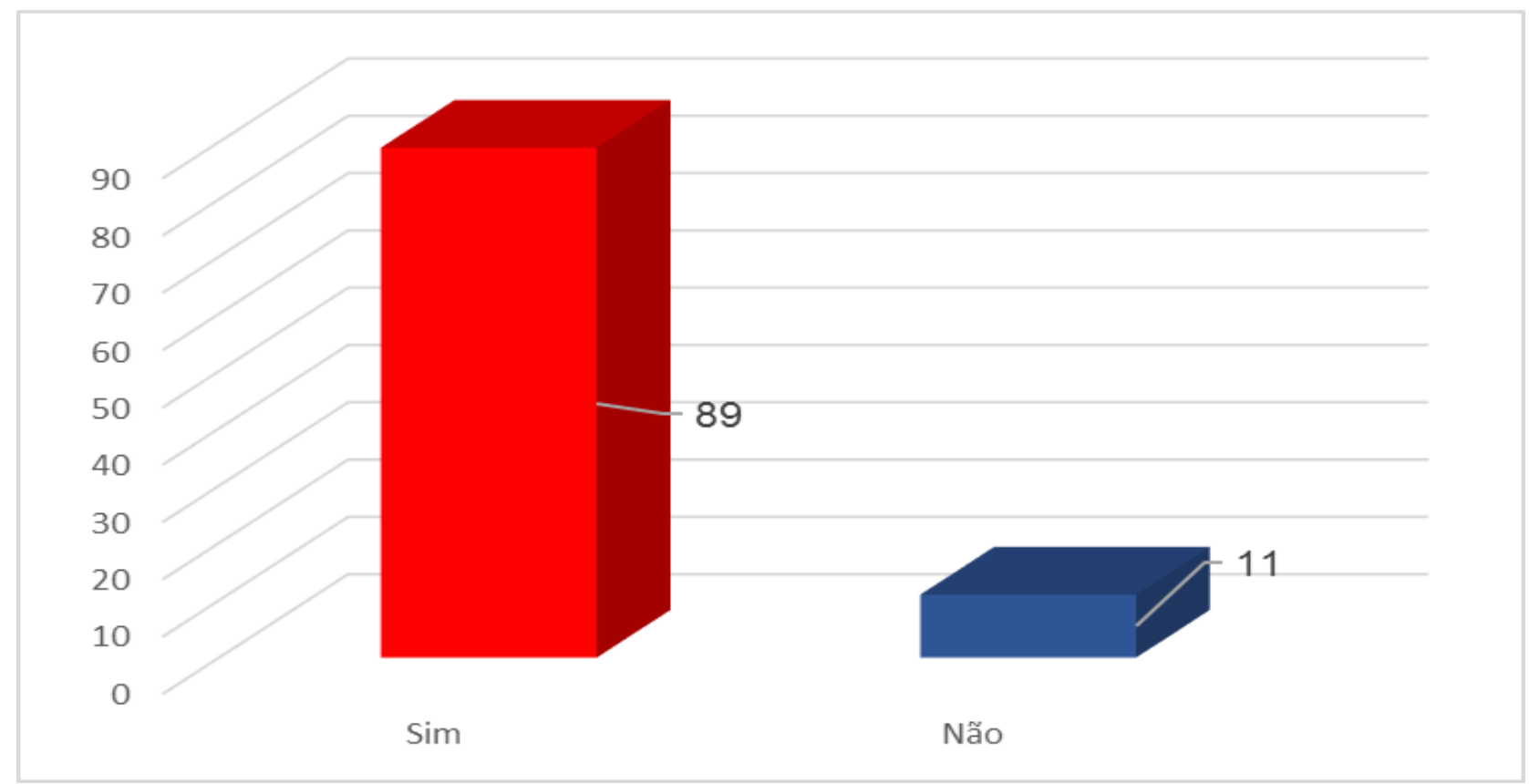

Fonte: Silva JO Oliveira ME e Oliveira TS, 2019.

Verifica-se na figura 2, em relação ao conhecimento dos enfermeiros sobre os parâmetros do ventilador mecânico, um percentual de $65 \%(n=12)$ dos enfermeiros que relataram possuir conhecimento sobre esses parâmetros e $35 \%(n=8)$ dos participantes afirmam não ter o entendimento dos parâmetros do ventilador mecânico.

Figura 2 - Distribuição das respostas dos Enfermeiros quanto ao conhecimento dos parâmetros do Ventilador Mecânico, Porto Velho, 2019.

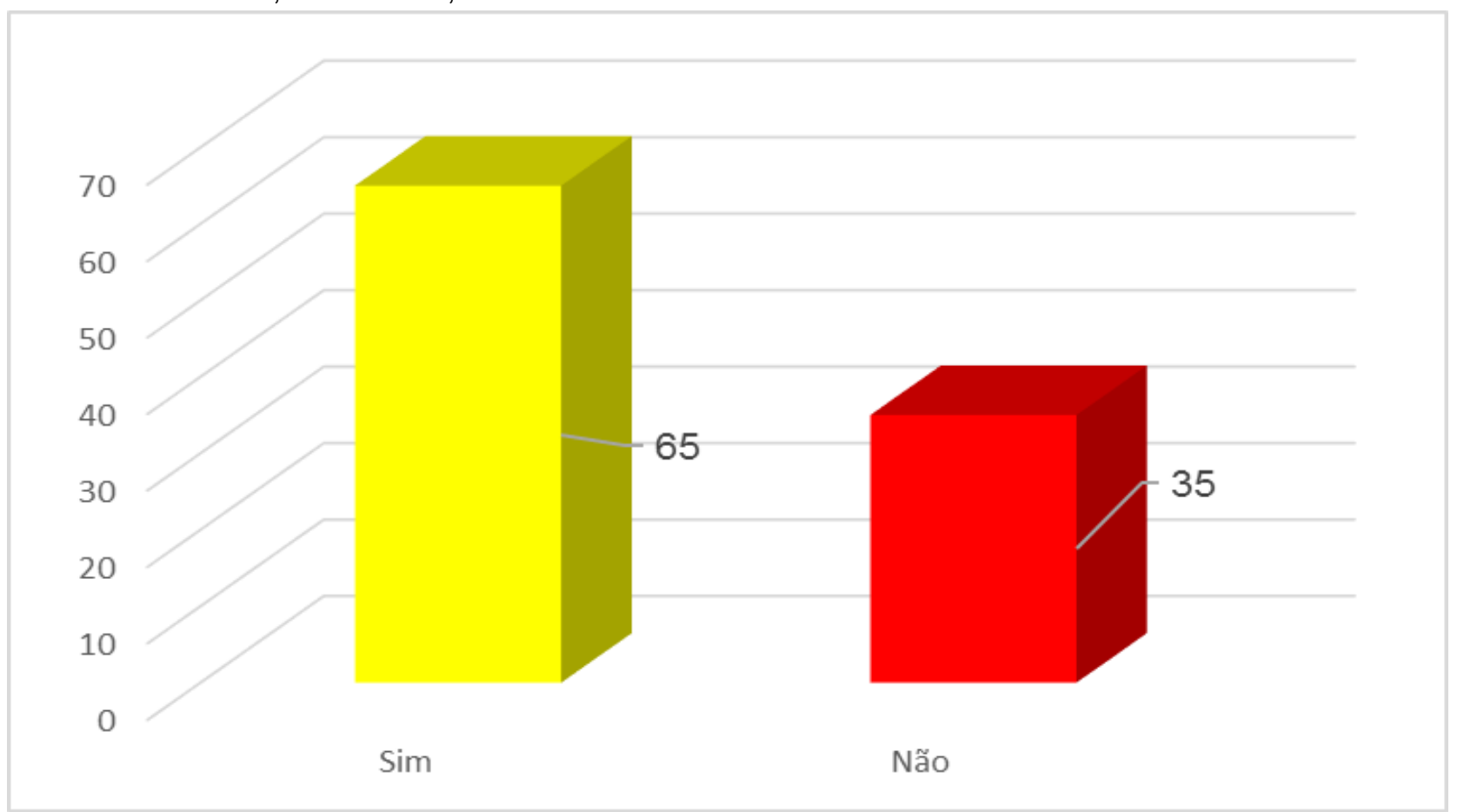

Fonte: Silva JO Oliveira ME e Oliveira TS, 2019. 
Desse modo, a Figura 3 retrata sobre a realização do preparo e montagem do Ventilador Mecânico em relação as respostas dos enfermeiros, foi evidenciando que $95 \%(n=18)$ dos participantes responderam que realizam esses procedimentos e $5 \%(n=2)$ relataram que não realiza esse preparo e montagem do VM.

Figura 3 - Distribuição das respostas dos Enfermeiros quanto a realização do preparo e montagem do VM, Porto Velho, 2019.



Fonte: Silva JO Oliveira ME e Oliveira TS, 2019.

No que tange a figura 4 evidencia que $85 \%(n=16)$ dos enfermeiros responderam realizar a programação dos parâmetros do Ventilador Mecânico dos pacientes sob VM e 15\% ( $n=4)$ afirmaram não realizar esse tipo de procedimento.

Figura 4 - Distribuição das respostas dos Enfermeiros sobre a programação dos parâmetros do Ventilador Mecânico dos pacientes sob VM, Porto Velho, 2019.

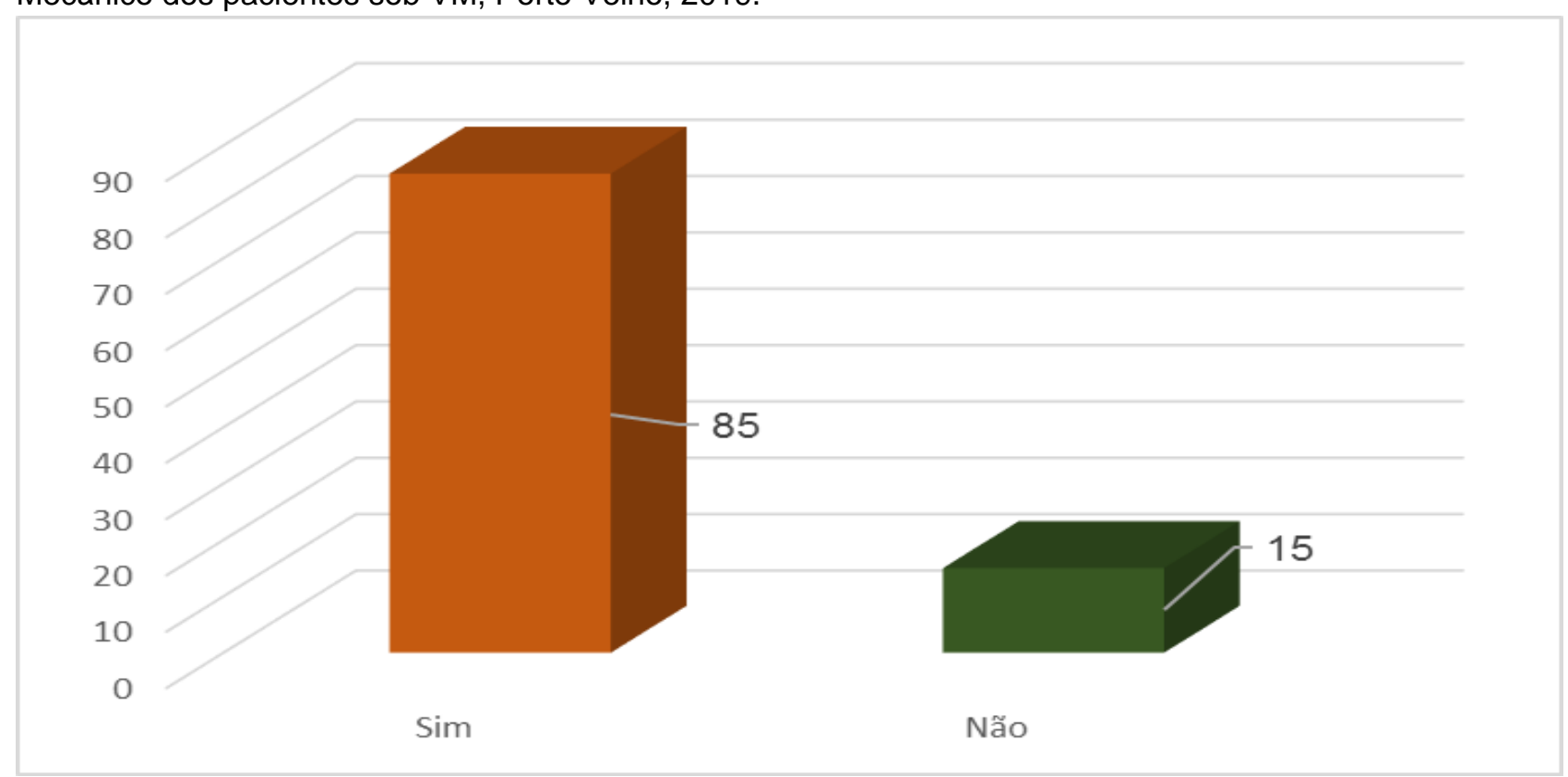

Fonte: Silva JO Oliveira ME e Oliveira TS, 2019. 
Em referência aos cuidados de enfermagem ao paciente sob VM os enfermeiros relataram que as seguintes respostas foram $57 \%(n=10)$ montagem e preparo do VM, 32\% $(n=6)$ para os alarmes do ventilador e por fim o percentual de $11 \%(n=3)$ para aspiração de secreções pulmonares. Todos estes dados explanados na Figura 5.

Figura 5 - Distribuição das respostas dos Enfermeiros sobre aos cuidados de enfermagem ao paciente sob VM, Porto Velho, 2019.

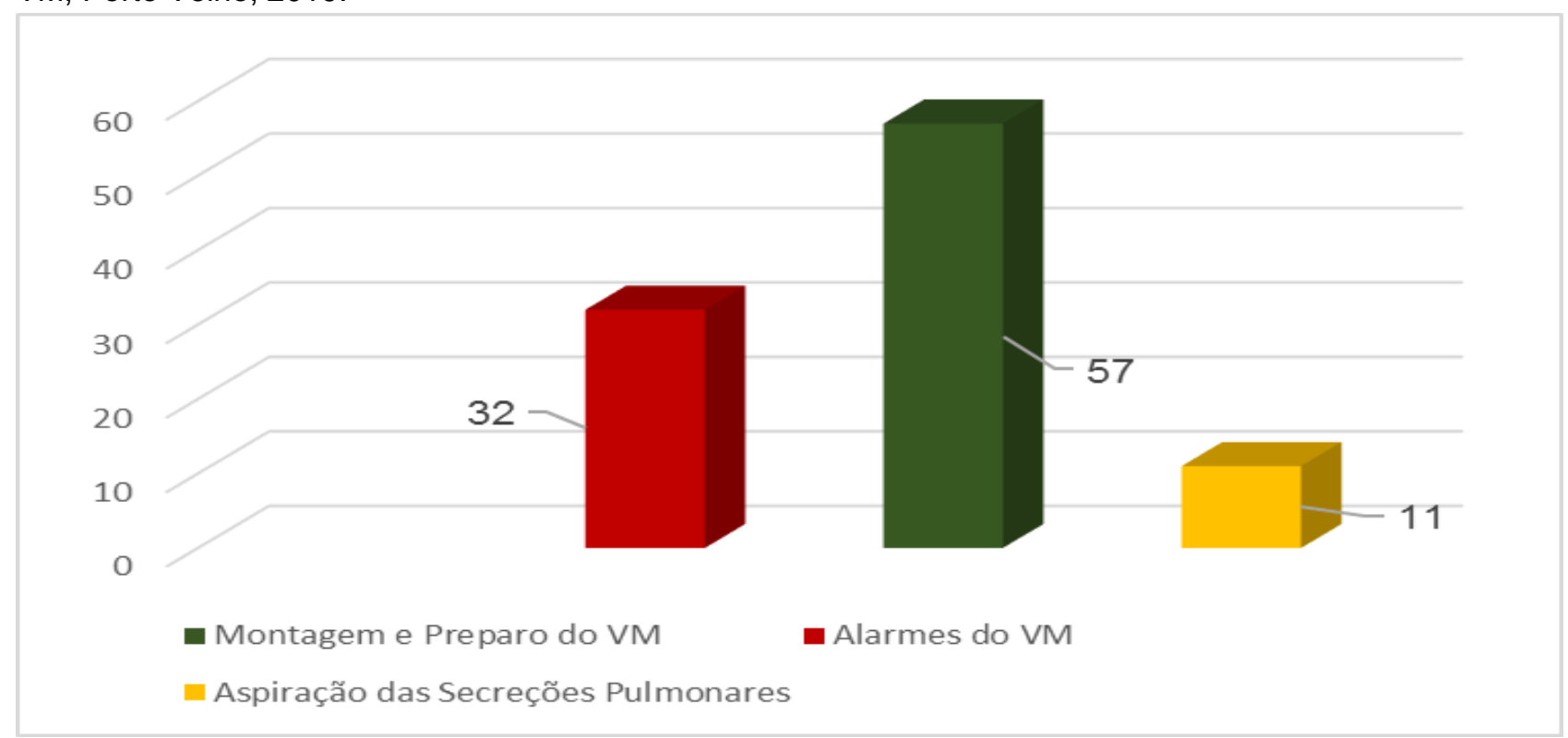

Fonte: Silva JO Oliveira ME e Oliveira TS, 2019.

\section{DISCUSSÃO}

Perez L (2014) destacou em seu estudo, o qual foi realizado em seis hospitais de Ribeirão Preto, São Paulo que dos 49 profissionais de enfermagem pesquisados, 47 eram do sexo feminino. Souza VS et al. (2015), também confirmam relatando que em três unidades de ensino do sul do Brasil, 47 dos 71 profissionais de enfermagem também eram do sexo feminino.

Pedrosa DF (2016), afirma em seu estudo que a enfermagem, por ser uma profissão de predomínio feminino, confere à mulher a oportunidade de gerenciar, não sendo comuns em nossos meios profissionais questionamentos sobre a capacidade administrativa das mulheres nesta área.

Na pesquisa obtivemos uma predominância de profissionais jovens com a faixa etária de 26 a 33 anos alcançando o maior índice totalizando $48 \%(n=9)$. Esta pesquisa corrobora com a de Freitas SD (2013) que em relação à idade dos participantes, houve uma variação de 26 a 35 anos ou mais, perfazendo uma média de 32 anos, mostrando a prevalência de uma força de trabalho jovem, com toda a capacidade produtiva.

No que se refere no estudo de Sampaio MS (2017), à faixa etária, destaca-se de 28 a 35 anos com (58\%), seguido dos profissionais jovens com idade entre 18 e 30 anos com (37,5\%). Outros estudos no mesmo seguimento reafirmam taxas elevadas nessa faixa etária, apontando um percentual de $53,8 \%$ até os 35 anos de idade e $46,2 \%$ acima dos 40 anos de idade.

No estudo de Ferreira SD (2015) em relação à titulação, apenas doze (51\%) tinham especialização, cinco (11\%), mestrado e (3\%) apresentava doutorado. Em relação à formação do enfermeiro, há que se diferenciar a formação acadêmica universitária, dando lugar à obtenção do título oficial de graduação em enfermagem, para a prática da profissão no sistema de saúde, e a formação acadêmica de pós-graduação, que inclui formação universitária em diferentes níveis acadêmicos, (Mestrado, Doutorado) e cursos de formação e atualização, assim como, eventos científicos (PEREZ L, 2014). 
Damasceno FD et al. (2015) afirmam que o enfermeiro com treinamento em UTI exerce suas funções sendo capacitados e qualificados nesse atendimento aos pacientes com VM, no entanto, nota-se que os enfermeiros, estão mais distantes do suporte ventilatório, possivelmente pelas múltiplas atribuições que lhes são atribuídas, ou por haver outra categoria profissional implementando esse tipo de assistência, assim como pela deficiência da sua percepção destas ações.

Nessa visão, os treinamentos para atuação na UTI devem ser compreendidos como um nível avançado e específico de capacitação/qualificação profissional em sentido estrito, incluindo o preparo para uma assistência de melhor qualidade possível proporcionado aos pacientes sob ventilação mecânica um cuidado fidedigno e de qualidade, com uma assistência continuada através do enfermeiro (LEITE FG, 2014).

Pelo exposto, é fundamental a importância que o enfermeiro se capacite e qualifique-se em cursos sobre o assunto abordado, de maneira a garantir o atendimento destes e dos demais pacientes com uma assistência adequada proporcionado aos pacientes sob ventilação mecânica um cuidado fidedigno e de qualidade (TAVARES EG et al., 2012).

No estudo de Frota NM (2014) e Rodrigues CDA et al. (2016) afirmam que os enfermeiros são capacitados e preparados para efetuar o cuidado de maneira sistemática e humanizada ao paciente em uso de VM.

Palhares MS (2016) diz que os cuidados de enfermagem mais citados se referem a aspiração de secreções pulmonares; observação dos alarmes do ventilador, cuidados com a manutenção do circuito e dispositivo do VM, manter e trocar a fixação do Tubo Orotraqueal (TOT) e traqueostomia (TQT)

Schwonke DF e Lunardi Filho QGP (2014) discorrem em seu estudo sobre os cuidados relacionados à aspiração das vias aéreas, em especial, sobre o uso do sistema fechado, destacando a relevância do conhecimento dos profissionais sobre a sua real importância ao assistir o paciente em VM e de executar esta técnica corretamente.

Os cuidados para a aspiração de secreções são descritos por Silva VFR e Torres AEPL (2016), esses autores acreditam que a aspiração de vias aéreas só deve ser feita quando necessário, evitando os soros de quaisquer tipos, garantindo a técnica asséptica e preferindo sempre o sistema fechado por atenuar os riscos de contaminação.

Para Gonçalves J et al. (2014), mostram em seu estudo o efeito da educação continuada dos enfermeiros, quando há intervenção na rotina de aspiração de secreções, que evidencia que a aspiração brônquica é um dos cuidados realizados com a técnica mais correta pelos profissionais.

Rodrigues CDA et al. (2016) observa em seu estudo que a aspiração brônquica é um dos cuidados mais citados pela enfermagem. A aspiração de secreções apesar de ser um cuidado muito conhecido pela enfermagem a ponto de ser um dos mais citados pelos profissionais, no que se refere ao paciente em VM, tem sua técnica colocada em dúvida quando percebemos que essa técnica é executada sem avaliação prévia do enfermeiro da real necessidade e muitas vezes de maneira equivocada.

Os alarmes dos ventiladores mecânicos são essenciais, pois apontam o progresso de complicações, de um suporte usado para manter a vida, portanto, à identificação da causa dos alarmes ventilatórios e a manutenção adequada dessa terapia são prioritárias, para melhorar a qualidade da assistência realizada (FARIAS FD, GUANE FGI, 2015).

Estudos mostram que os alarmes disparados por VM podem representar de 30 a $33 \%$ de todos os alarmes de uma unidade de cuidados intensivos, sendo os alarmes de elevação da frequência respiratória e de elevação da pressão de vias aéreas os mais prevalentes (PEDROSA DF 2016).

Os cuidados ao assistir o paciente em ventilação mecânica são infinitos, cada um deles antecipa a prevenção de que um risco em potencial não se torne um evento de verdade, dentre todos os cuidados, o de maior importância é o conhecimento (RODRIGUES CDA et al., 2016). É imprescindível que o profissional esteja sempre em vigilância, garantindo o cuidado e assim minimizando os riscos (MOLINARO LC, 2017). 
O enfermeiro necessita de competência técnica e interpessoal com um paciente em ventilação mecânica, precisa perceber as mudanças na situação clínica dos pacientes, decifrar e interceder corretamente, a ventilação mecânica é intensiva, extensa, complexa e engloba múltiplos procedimentos, entre eles estão: controle de sinais vitais, monitorização cardiovascular, monitorização das trocas gasosas e de padrão respiratório e vigilância constante (SILVA LCC, 2015).

O enfermeiro atuando nessa unidade pode analisar e apontar e propor à equipe multiprofissional a provável necessidade do procedimento de sedação, quando beneficia ao cliente. A evolução dessa terapia está exatamente associada ao padrão respiratório e à oxigenação correta sendo individual a cada paciente (PEDROSA DF, 2016).

\section{CONCLUSÃO}

Desta forma, acredita-se na importância de avaliar o conhecimento do enfermeiro que lida constantemente com os pacientes críticos em uso de ventilação mecânica e enfatiza-se que a assistência demanda um conhecimento básico, no mínimo, para intervir junto à equipe sempre que necessário. Esperase que esta pesquisa contribua com novos estudos acerca da qualificação/atualização do profissional enfermeiro a fim de que novas estratégias de assistência sejam alcançadas.

\section{REFERÊNCIAS}

1. CARRARETTO AR. Ventilação pulmonar em anestesia adulta. Curso de Educação à Distância em Anestesiologia. São Paulo: 2014.

2. DAMASCENO FD et al. Fundamentos de Enfermagem em Cuidados Críticos da AACN. Porto alegre: Artmed, 2015.

3. FARIAS FD; GUANES FGI. Fatores associados à maior mortalidade e tempo de internação prolongado em uma unidade de terapia intensiva de adultos. Rev bras ter intensiva. v.22, n.3, pp.250-256. São Paulo. 2015.

4. FERREIRA SD. Ventilação Mecânica: avaliando o conhecimento dos enfermeiros. Ágora: Rev Divulg. Cient., Mafra, v. 16, n. 2, 2015.

5. FREITAS SD. Sistema de apoio à decisão na monitorização do paciente em assistência ventilatória invasiva. 2013 . Dissertação (Mestrado em Tecnologia em Saúde) - Centro de Ciências Biológicas e da Saúde, Pontifícia Universidade Católica do Paraná, Curitiba, 2013.

6. FROTA NM. Assistência de enfermagem ao paciente sob ventilação mecânica internado em unidade de terapia intensiva. Revista Gaúcha de Enfermagem. v.1 n. 2. Mar-abr. São Paulo. 2014.

7. GONÇALVES J et al. Ventilação Mecânica: Evidências Para o Cuidado de Enfermagem. Esc Anna Nery. v.16, n.4, pp.789-795. Rio de Janeiro. 2014.

8. LEITE FG. Caracterização do estresse nos enfermeiros de unidades de terapia intensiva. Revista Escola de Enfermagem USP. v.2, n.3 fev-mar. São Paulo. 2014.

9. NÓBREGA MML de. Conhecimento na Enfermagem na Ventilação Mecânica. Revista Nursing-Edição Brasileira: Editorial Bolina Brasil, n. 110, p.293I, jul. 2014.

10. PEREZ L. Situação do Sistema de Saúde no Brasil e os Cuidados Desenvolvidos nas Unidades de Terapia Intensiva. Revista de Enfermagem EEAN/UFRJ; Rio de Janeiro, v. 5, n. 2, p. 259-61, Rio de Janeiro. 2014.

11. PEDROSA DF Ventilação mecânica não-invasiva com pressão positiva. Revista Brasileira de Terapia Intensiva, v. 19, n. 2, São Paulo. 2016.

12. POLKEY E; MOXHAM DF. Enfermagem em terapia intensiva. São Paulo: Atheneu. 2015.

13. RODRIGUES CDA et al. Ventilação mecânica. São Paulo: Atheneu; 2016.

14. SAMPAIO MS. Comunicação: Equipe de Enfermagem e Paciente em Ventilação Mecânica. R. Enferm. UFSM. 2011 Set/Dez. 2017.

15. SILVA VFR; TORRES AEPL. Intervenção e atividades propostas para o diagnóstico de enfermagem - ventilação espontânea prejudicada. Revista Acta Paulista. Rio de Janeiro. 2016.

16. SILVA LCC da. Condutas em Pneumologia. Rio de Janeiro: Revinter, 2015.

17. SCHWONKE DF; LUNARDI FILHO QGP. Ventilação mecânica básica para enfermagem. São Paulo: Atheneu, 2014.

18. SOUZA VS et al. Erros e eventos adversos: A interface com a cultura de segurança dos profissionais de saúde. Revista Cogitare Enfermagem. v. 20, n.3, p. 475-482. Paraná, 2015.

19. TAVARES EG. Cuidados de Enfermagem ao Cliente em Uso de Ventilação Mecânica Invasiva. Revista Latinoamericana de Enfermagem. Série IV - n. ${ }^{\circ} 1$ - Fev./Mar. São Paulo. 2012. 